\title{
Wise Anaerobic Work Station: an evaluation
}

\author{
PENELOPE R SISSON, ${ }^{*}$ H R INGHAM, ${ }^{*}$ P O BYRNE† \\ From the *Microbiology Department and the †Regional Medical Physics Department, The General Hospital, \\ Newcastle upon Tyne
}

SUMmaRY The Wise Anaerobic Work Station permitted the growth of type cultures of a range of fastidious obligate anaerobes. In the isolation of anaerobic bacteria from clinical specimens the performance equalled that of a standard anaerobic jar incubated for 48 hours, and gave results superior to those obtained when incubation of jar cultures was interrupted at 24 hours. The Wise Anaerobic Work Station was easy to use and provided comfortable working conditions; running largely on industrial nitrogen, it was more economic than anaerobic cabinets or jars using conventional gas mixtures.

Since their introduction in the late 1970's anaerobic cabinets have become more complex and expensive, with little objective evidence of increasing effectiveness. The models currently available include daunting control panels and instruction manuals running to several volumes, but operators find that only a minority of the switches and dials are used. The impact that such cabinets have had on the standard of anaerobic microbiology practised is hard to quantify, but demand for their inclusion in the inventory of any microbiology department cannot be ignored.

Don Whitley Scientific Ltd manufacture several anaerobic cabinets, and the latest addition to their range, the Wise Anaerobic Work Station, is a simple cabinet with little high technology refinement. The Wise apparatus incorporates a primary chamber flushed with nitrogen in place of the conventional lock using anaerobic gas mixture $(10 \%$ hydrogen, $10 \%$ carbon dioxide, and $80 \%$ nitrogen) and is designed to reduce substantially the running costs.

We report here an evaluation of the effectiveness of the Wise Anaerobic Work Station, with particular reference to the achievement and maintenance of anaerobiosis, ease of operation, and running costs.

\section{Material and methods}

The Wise Anaerobic Work Station (Don Whitley Scientific Ltd) comprises two chambers and a pass box made of acrylic plastic with glove ports in each chamber (fig 1). The pass box leads into a chamber flushed with industrial nitrogen (BOC Ltd) in which cultures are vented before being transferred to the Accepted for publication 24 September 1986 anaerobic chamber containing $10 \%$ hydrogen, $10 \%$ carbon dioxide, and $80 \%$ nitrogen (BOC). The ports to the nitrogen chamber have open ended gloves enabling the operator to work barehanded within the chamber; access to the anaerobic chamber is via full gloves. There is also a facility that allows single plates to be passed directly into the anaerobic atmosphere without pre-gassing in the nitrogen chamber (single plate port). There is an oil bubble bottle system to equalise the pressures in the two chambers and a second bubble bottle allowing excess pressure in the nitrogen chamber to be vented to the atmosphere. Both chambers are fitted with a catalyst sachet, which is situated in front of the heater in the anaerobic chamber so that it is kept dry. Each chamber has a manually switched cooling plate (fig 1) that condenses water from the chamber's atmosphere, eliminating the need for a humidistat.

\section{B ACTERIA}

Clostridium tetani (NCTC strains 2938, 3795, and 9567), $C$ novyi type D (NCTC strains 8350 and 9692), Bacteroides fragilis (NCTC strain 8560), B asaccharolyticus isolated from a clinical specimen, and Pseudomonas aeruginosa (NCTC strain 19662) were used in the evaluation of the cabinet. Organisms were maintained by weekly subculture on $5 \%$ horse blood agar plates, with the exception of the strains of $C$ novyi, which were grown on blood agar plates supplemented with $1 \mathrm{~g} / \mathrm{l}$ cysteine $+90 \mathrm{mg} / 1$ dithiothreitol (CDA). The obligate anaerobes were maintained in standard anaerobic jars fitted with a cold palladium catalyst (Don Whitley Scientific Ltd) in an atmosphere of $90 \%$ hydrogen and $10 \%$ carbon dioxide. 


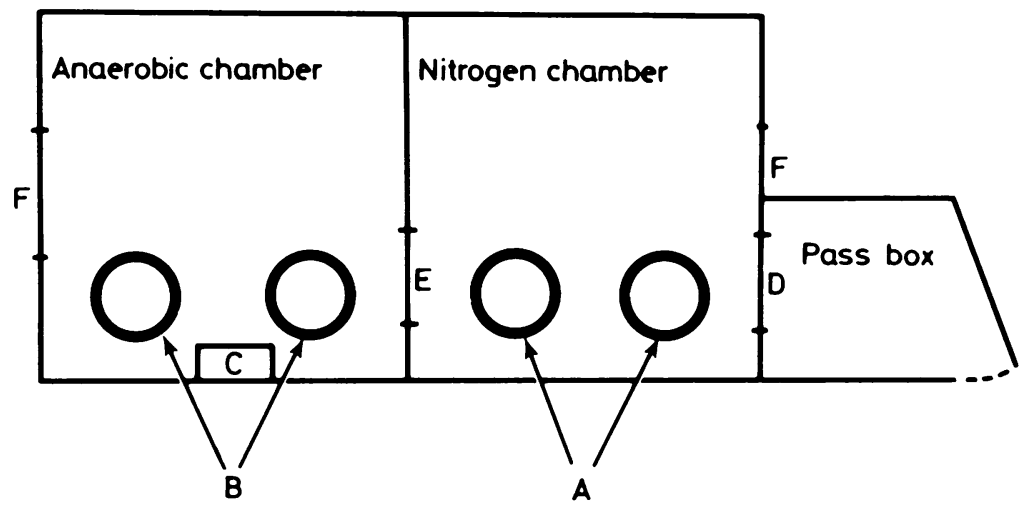

Fig 1 Diagrammatic representation of Wise Anaerobic Work Station

\section{REDOX AND $\mathrm{PO}_{2}$ CONCENTRATIONS}

The Wise cabinet was modified to allow cables to be passed into both chambers so that physical measurements could be made in situ. The redox and $\mathrm{pH}$ measurements were made with a Beckman 3500 digital $\mathrm{pH}$ meter in conjunction with probes supplied by Russell pH Ltd. The pH probe was calibrated with standard pH solutions (BDH Chemicals Ltd), and the redox electrode was standardised against a $0 \cdot 1 \mathrm{M}$ ferrous-ferric ammonium sulphate solution in $\mathbf{M}$ $\mathrm{H}_{2} \mathrm{SO}_{4}$, which has a redox of $476 \mathrm{mV}^{1}$.

Measurements of $\mathrm{pO}_{2}$ values were made with a $\mathrm{pO}_{2}$ probe supplied by Russell pH Ltd. The probe was calibrated, as recommended by the manufacturers, on the basis that $20.9 \%$ of the atmosphere consists of oxygen. In initial studies attempts to obtain $\mathrm{pO}_{2}$ values in liquid media presented technical problems due to oxygen consumption across the membrane and the lack of appropriate maximum concentration liquid standards; although not further pursued here, it will form the subject of a further communication.

\section{GROWTH OF ANAEROBES}

All strains were inoculated from a single colony of a pure culture on to three sets of blood agar plates or

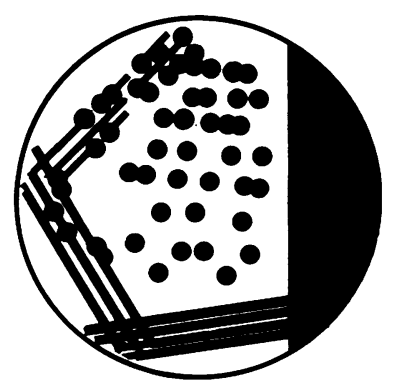

Fig 2 Schematic diagram showing distribution of colonies resulting from plating method
CDA as appropriate (fig 2). One set of plates was incubated in the anaerobic chamber, the second set in a normal incubator (aerobic control) and the third a conventional anaerobic jar (anaerobic control) all at $37^{\circ} \mathrm{C}$. All plates were scored for growth at 24 and 48 hours.

\section{SURVIVAL OF ANAEROBES IN NITROGEN} ENVIRONMENT

All strains were inoculated as above on to 20 sets of plates; four were distributed as above, and eight further sets were initially placed in the nitrogen chamber. Subsequently, one set of plates from the nitrogen chamber was transferred to the anaerobic chamber every day and a second (control set) was transferred to an anaerobic jar. Similarly, the other eight sets were left in air initially, and, daily, one set was transferred to the anaerobic chamber and one set to the anaerobic jar. All plates were scored for growth at 24 and 48 hours semiquantatively: ++ represented luxuriant growth; + growth of a roughly equal number of colonies as that of the control but of diminished colony size; and \pm growth of $\leqslant 20$ colonies per plate.

\section{REDOX POTENTIAL}

Sequential redox potential and concurrent $\mathrm{pH}$ measurement were made in nutrient broth in both chambers and aerobically when the cabinet was left unused, and before and after various manipulations.

Measurements of $\mathrm{pO}_{2}$ were similarly made of the atmosphere in both chambers and compared with those obtained in air.

\section{GROWTH OF TEST ANAEROBES IN NITROGEN ENVIRONMENT}

Preliminary experiments indicated very low $\mathrm{pO}_{2}$ values in the nitrogen chamber, and in the absence of a heating element in this chamber the following experiment was devised to determine the ability of the 
Table 1 Survival of test anaerobes in the nitrogen chamber

\begin{tabular}{|c|c|c|c|c|c|c|c|c|}
\hline & \multicolumn{2}{|c|}{ One day in: } & \multicolumn{2}{|c|}{ Two days in: } & \multicolumn{2}{|c|}{ Three days in: } & \multicolumn{2}{|c|}{ Four days in: } \\
\hline & Nitrogen & Air & Nitrogen & Air & Nitrogen & Air & Nitrogen & Air \\
\hline $\begin{array}{l}\text { C tetani } \\
\text { (NCTC 9567) }\end{array}$ & + & \pm & + & \pm & + & \pm & + & \pm \\
\hline $\begin{array}{l}\text { C tetani } \\
\text { (NCTC 2938) }\end{array}$ & + & + & + & + & + & \pm & + & \pm \\
\hline $\begin{array}{l}\text { C tetani } \\
\text { (NCTC 3795) }\end{array}$ & + & + & + & + & + & \pm & + & \pm \\
\hline $\begin{array}{l}\text { C novyi } \\
\text { (NCTC 9692) }\end{array}$ & NG & NG & NG & NG & NG & NG & NG & NG \\
\hline $\begin{array}{l}\text { C novyi } \\
\text { (NCTC 8350) }\end{array}$ & NG & NG & NG & NG & NG & NG & NG & NG \\
\hline $\begin{array}{l}\text { B fragilis } \\
\text { (NCTC 8569) }\end{array}$ & ++ & ++ & ++ & ++ & ++ & ++ & ++ & ++ \\
\hline$B$ asaccharolyticus & ++ & + & $+t$ & \pm & ++ & \pm & ++ & NG \\
\hline
\end{tabular}

$++=$ luxuriant growth; $+=$ growth of a roughly equivalent number of colonies but with reduced individual colony size; $\pm=$ growth of $\leqslant 20$ colonies; $\mathrm{NG}=$ no growth.

nitrogen atmosphere to support growth of obligate anaerobes. Blood agar slopes were prepared in universal containers that were pre-exposed to nitrogen for three hours to allow equilibration before the organisms were inoculated; the atmosphere was retained by simply securing the caps, and bottles were then transferred to an incubator at $37^{\circ} \mathrm{C}$. Appropriate controls with pre-exposure to, or incubation in, anaerobic or aerobic conditions were run simultaneously.

COMPARISON OF YIELD OF OBLIGATE ANAEROBES FROM CLINICAL SPECIMENS

Clinical specimens processed in the laboratory over three weeks were also cultured on duplicate blood agar plates, one set of which was incubated in anaerobic jars and the second in the Wise Anaerobic Work Station. They were examined at 48 hours, and all colonial types not represented on the corresponding routine aerobic culture were subcultured on blood agar plates incubated aerobically and anaerobically. A metronidazole disc $(5 \mu \mathrm{g})$ was placed on the second streak of the anaerobic subculture, and any organism inhibited by this and failing to grow aerobically was designated an obligate anaerobe. Subsequent charac- terisation was performed according to the schemes of Duerden $e t a l^{2}$ and Cowan and Steel. ${ }^{3}$ Retrospectively, the number of anaerobes isolated by our routine procedure of culturing specimens on blood agar plates incubated in standard anaerobic jars opened at 24 hours and 48 hours was compared with uninterrupted anaerobic incubation in anaerobic jars and the Wise cabinet.

\section{Results}

The method in which plates were inoculated produces about 50 discrete colonies in the centre of the plate (fig 2 ), which readily allows comparison of growth under different conditions.

The anaerobic chamber of the Wise cabinet allowed growth of all the test anaerobes. Growth of $C$ tetani, $B$ fragilis, and $B$ asaccharolyticus was readily apparent at 24 hours while strains of $C$ novyi required 48 hours' incubation for visible growth. In all instances the growth was equivalent to that on the control plates incubated in standard anaerobic conditions. There was occasionally a minimal discernible growth of Pseudomonas aeruginosa on blood agar plates after 24 hours' incubation in the anaerobic

Table 2 Sequential redox and pH measurements in nutrient broth* in nitrogen and anaerobic chambers

\begin{tabular}{|c|c|c|c|c|}
\hline \multirow{2}{*}{$\begin{array}{l}\text { Time } \\
\text { (hours) }\end{array}$} & \multicolumn{2}{|c|}{ Nitrogen chamber } & \multicolumn{2}{|c|}{ Anaerobic chamber } \\
\hline & $\operatorname{Redox}(\mathrm{m} V)$ & $p H$ & $\operatorname{Redox}(m V)$ & $p H$ \\
\hline $\begin{array}{l}T_{0} \\
T_{1} \\
T_{2} \\
T_{3}^{3} \\
T_{4} \\
T_{5} \\
T_{24}\end{array}$ & $\begin{array}{r}41 \\
51 \\
-\quad 27 \\
-106 \\
-263 \\
-305 \\
-299\end{array}$ & $\begin{array}{l}7 \cdot 2 \\
7 \cdot 2 \\
7 \cdot 2 \\
7 \cdot 3 \\
7 \cdot 2 \\
7 \cdot 2 \\
7 \cdot 2\end{array}$ & $\begin{array}{r}45 \\
10 \\
-71 \\
-260 \\
-325 \\
-539 \\
-544\end{array}$ & $\begin{array}{l}7 \cdot 3 \\
7 \cdot 3 \\
7 \cdot 2 \\
7 \cdot 3 \\
7 \cdot 3 \\
7 \cdot 1 \\
7 \cdot 1\end{array}$ \\
\hline
\end{tabular}

*Broths left unshaken. 
Table 3 Growth of test anaerobes in nitrogen environment

\begin{tabular}{|c|c|c|c|c|c|}
\hline \multirow[b]{2}{*}{ Strain } & \multicolumn{5}{|l|}{ Prereduction } \\
\hline & Anaerobic ${ }^{*}$ & Nitrogen* & Air $^{*}$ & Anaerobic $^{+}$ & Anaerobic $^{++}$ \\
\hline \multirow{5}{*}{$\begin{array}{l}\text { B fragilis } \\
\text { (NCTC 8560) } \\
\text { B asaccharolyticus } \\
\text { C tetani } \\
\text { (NCTC 2938) } \\
\text { C tetani } \\
\text { (NCTC 3795) } \\
\text { C tetani } \\
\text { (NCTC 9567) } \\
\text { Ps aeruginosa } \\
\text { (NCTC 19662) }\end{array}$} & ++ & ++ & NG & ++ & ++ \\
\hline & $\stackrel{+}{\text { NG }}$ & $\stackrel{+}{\text { NG }}$ & $\begin{array}{l}\text { NG } \\
\text { NG }\end{array}$ & $\begin{array}{l}++ \\
++\end{array}$ & $\begin{array}{l}+ \\
+\end{array}$ \\
\hline & NG & NG & NG & ++ & + \\
\hline & + & + & NG & ++ & ++ \\
\hline & NG & \pm & \pm & NG & NG \\
\hline
\end{tabular}

*Slopes inoculated in nitrogen chamber, lids tightened, incubated in aerobic incubator; ${ }^{+}$slopes inoculated in nitrogen chamber, returned to anaerobic chamber, incubated without lids; ${ }^{+}+$slopes inoculated in nitrogen chamber, returned to anaerobic chamber, lids tightened, slopes incubated in aerobic incubator.

chamber, whereas there was none on a similar plate incubated in an anaerobic jar.

None of the test anaerobes grew in the unheated nitrogen side of the cabinet but there was luxuriant growth of $P$ s aeruginosa after 24 hours.

Table 1 shows the results of the survival experiment. All strains other than $C$ novyi survived four days' exposure to nitrogen when inoculated on blood agar plates and subsequently incubated; but all the strains, with the exception of $B$ asaccharolyticus, also survived exposure to air for the same period of time. Less than 20 colonies of $C$ tetani, however, grew on plates exposed to air, possibly representing spores surviving exposure to oxygen, whereas the vegetative forms succumbed. In contrast, the counts of $C$ tetani on the plates exposed to nitrogen for four days were similar to those of the control plates, suggesting that the vegetative forms remained viable.

Sequential redox and $\mathrm{pH}$ measurements of nutrient broths introduced into each chamber were made at least thrice; table 2 shows the typical results. Thus after about five hours the redox of the broth incubated anaerobically was less than $-500 \mathrm{mV}$ compared with $-305 \mathrm{mV}$ for the broth left in the nitrogen chamber.

The effect of opening various ports for controlled times on redox and $\mathrm{pH}$ was investigated and in no instance was any noticeable perturbation noted. For instance, seven nutrient broths left overnight in the anaerobic chamber in various locations all had redox potentials of about $-580 \mathrm{mV}(\mathrm{pH} 7 \cdot 1)$ which remained unchanged after the single plate port was used seven times in quick succession.

The effect of pre-reducing the media on its ability to support the growth of obligate anaerobes in various atmospheres was investigated using blood agar slopes (table 3). Prereduction in the nitrogen chamber for three hours with subsequent incubation in nitrogen permitted the growth of $B$ fragilis, $B$ asaccharolyticus, and one strain of $C$ tetani, as did prereduction anaerobically followed by incubation in nitrogen. By contrast, no organism grew in nitrogen on media that had not been pre-reduced. Prereduction in the anaerobic side of the chamber permitted the growth of all anaerobes studied in this experiment, whether incu-

Table 4 Obligate anaerobes isolated from 236 specimens cultured in Wise Anaerobic Work Station or standard anaerobic jars for 48 hours

No of isolates using:

\begin{tabular}{lrr}
\cline { 2 - 3 } Bacteria & Wise & Anaerobic jar \\
\hline Anaerobic Gram positive coccit & 124 & 100 \\
Veillonella & 6 & 2 \\
B melaninogenicus* & 25 & 19 \\
B fragilis & 16 & 13 \\
$B$ uniformis & 1 & 0 \\
B corrodens & 3 & 4 \\
Fusobacterium spp & 3 & 1 \\
Clostridium spp & 3 & 1 \\
Total & 181 & 140 \\
\hline
\end{tabular}

*Including $B$ asaccharolyticus, $B$ intermedius, and B melaninogenicus; tgrow only in anaerobic conditions and sensitive to metronidazole (5 $\mu \mathrm{g})$. 
Table 5 Obligate anaerobes isolated from 185 specimens cultured in Wise Anaerobic Work Station for 48 hours, or standard anaerobic jars*

\begin{tabular}{|c|c|c|c|}
\hline \multirow[b]{2}{*}{ Bacteria } & \multicolumn{3}{|c|}{ No of isolates using: } \\
\hline & Wise & $\begin{array}{l}\text { Anaerobic jar } \\
\text { ( } 48 \text { hours continuous })\end{array}$ & $\begin{array}{l}\text { Anaerobic jar } \\
\text { ( } 48 \text { hours discontinuous })\end{array}$ \\
\hline Anaerobic streptococcus & 76 & 60 & 16 \\
\hline Veillonella & 5 & 2 & 0 \\
\hline B melaninogenicust & 14 & 12 & 2 \\
\hline$B$ fragilis & 11 & 10 & 8 \\
\hline$B$ uniformis & 1 & 0 & 1 \\
\hline$B$ corrodens & $i$ & 2 & 0 \\
\hline Fusobacterium spp & 1 & 1 & 0 \\
\hline Clostridium spp & 3 & 1 & 0 \\
\hline Total & 112 & 88 & 27 \\
\hline
\end{tabular}

*Either unopened for 48 hours or opened at 24 hours and reincubated for a further 24 hours.

tincluding $B$ asaccharolyticus, $B$ intermedius, and $B$ melaninogenicus.

bated in the anaerobic side of the cabinet with the lids off, or in aerobic incubators with the lids screwed on.

A total of 236 clinical specimens were cultured, 103 $(43.6 \%)$ of which yielded anaerobes when incubated in the Wise Anaerobic Work Station, compared with $82(34.7 \%)$ when cultured in standard anaerobic jars for 48 hours. Table 4 shows the numbers and types of anaerobes isolated by the two methods. Of the 236 specimens, 51 were high vaginal swabs, which are not reincubated anaerobically beyond 24 hours during the routine procedure. Table 5 shows the anaerobes isolated from the remaining 185 specimens cultured by these procedures. The routine culture method yielded anaerobes from only 18 of 185 specimens $(9 \cdot 7 \%)$ compared with $52(28.1 \%)$ incubated in anaerobic jars unopened for 48 hours and $66(35 \cdot 7 \%)$ cultured in the Wise cabinet.

USE OF GAS

During the course of this evaluation use of the cabinet approximated that generated by the routine workload of this laboratory. The Wise cabinet required one anaerobic gas mixture cylinder about every six months and a cylinder of industrial nitrogen every three to four weeks.

\section{Discussion}

The Wise cabinet was comfortable and convenient to work in. The perspex panels allow good lighting and are much less claustrophobic than the opaque sides of the more conventional cabinets. The simplicity of the control panel was much appreciated and no loss of performance was detected-for instance, the use of an in situ thermometer presented no problem.

The condenser plates worked very effectively and when run for one to two hours per day, culture plates could be incubated inverted, without excessive water or condensation forming on the lids. The cabinet proved extremely economical in terms of running costs of the gases used. The nitrogen cylinder (about $£ 3$ on exchange from $\mathrm{BOC}$ ) lasted roughly one month under a substantial experimental workload at least equivalent to the use for routine specimens in this laboratory. The anaerobic gas mixture cylinder (about $£ 51$ on exchange from BOC) was sufficient for six months work. A Don Whitley Mk I cabinet elsewhere in this laboratory requires an anaerobic gas mixture cylinder about every month under a similar workload; with anaerobic jars the requirement is one cylinder every two months. This economy results from the use of nitrogen for flushing out most of the oxygen introduced with specimens. Much of this is contained in the air above Petri dishes or broths and is largely removed by venting specimen containers in the nitrogen chamber. Consequently, the only demand for anaerobic gas mixture is the top up required after either pass port has been opened to allow transfer of specimens into or out of the anaerobic chamber, and the very small amount required to replace the hydrogen catalysed to water in the process of removing residual oxygen.

The redox potential measurements show levels in the anaerobic chamber equivalent to those obtained in the best anaerobic jars, ${ }^{4}$ and the $\mathrm{pO}_{2}$ measurements confirm that very little oxygen remains in the atmosphere of either chamber. Removal of oxygen from the nitrogen chamber presumably arises because "spill-over" anaerobic gas mixture via the "bubblebottle" contains sufficient hydrogen to catalyse introduced oxygen.

Type cultures of a range of obligate anaerobes grew equally well in the Wise cabinet and standard anaerobic jars. The function of the nitrogen chamber seems to be largely that of purging oxygen; certainly there was little evidence that storage of inoculated plates 
improved the survival of the more fastidious organisms such as $C$ tetani and $C$ novyi.

The Wise cabinet compares very favourably with standard anaerobic jars when used for the culture of anaerobes from routine clinical specimens. Indeed, the excellent visibility and ease of use of the anaerobic cabinet facilitates examination of culture plates at 24 hours without interruption of anaerobiosis. Anaerobic culture of 185 of 236 specimens carried out by the routine procedure in our laboratory showed that only $9.7 \%$ of specimens cultured thus yielded anaerobes compared with $35.5 \%$ cultured in the Wise cabinet and $28 \cdot 1 \%$ cultured in anaerobic jars continuously for 48 hours. This confirms that uninterrupted anaerobic incubation for 48 hours substantially increases the yield of anaerobic organisms. ${ }^{5}$

Although previous anaerobic cabinets may have been regarded as valuable research equipment, ${ }^{6}$ the simplicity and economy of the Wise cabinet makes it eminently suitable as an anaerobic incubator for any microbiology department. It has proved reliable and convenient, and its running costs must compare favourably with those of conventional anaerobic jars whether they are gassed with anaerobic gas mixture or commercial packs generating hydrogen and carbon dioxide in appropriate concentrations.

We are grateful to Don Whitley Scientific Ltd for supplying the Wise Anaerobic Work Station, features of which are the subject of patent applications throughout the world, and to Miss S Appleby for typing the manuscript.

\section{References}

1 Light TS. Standard solution for redox potential measurements. Anal Chem 1972;44:1038-9.

2 Duerden BI, Collee JG, Brown R, Deacon AG, Holbrook WP. A scheme for the identification of clinical isolates of gramnegative anaerobic bacilli by conventional bacteriological tests. $J$ Med Microbiol 1980;13:231-45.

3 Cowan ST, Steel KJ. Manual for the identification of medical bacteria. 2nd edition. London: Cambridge University Press, 1974.

4 Ingham HR, Sisson PR, Eisenstadt RL, Sprott MS, Byrne PO, Selkon JB. Enhancement of activity of metronidazole by Escherichia coli under sub-optimal anaerobic conditions. J Antimicrob Chemother 1981;8:475-9.

5 Wren MWD. The culture of clinical specimens for anaerobic bacteria: a comparison of three regimens. J Med Microbiol 1977; 10:195-201.

6 Watt B, Collee JG, Brown R. The isolation of strict anaerobes: the use of an anaerobic cabinet compared with a conventional procedure. J Med Microbiol 1974;7:315-24.

Requests for reprints to: Dr HR Ingham, Consultant Microbiologist, The General Hospital, Westgate Road, Newcastle upon Tyne NE4 6BE, England. 